\title{
Efficacy and safety of adalimumab in paediatric patients with moderate-to-severe ulcerative colitis (ENVISION I): a randomised, controlled, phase 3 study
}

\author{
Nicholas M Croft, William A Faubion Jr, Subra Kugathasan, Jaroslaw Kierkus, Frank M Ruemmele, Toshiaki Shimizu, Nael M Mostafa, \\ Mary Venetucci, Tricia Finney-Hayward, Yuri Sanchez Gonzalez, Mareike Bereswill, Andreas Lazar, Dan Turner
}

\begin{abstract}
Summary
Background Biologic treatment options are limited for children with ulcerative colitis. The aim of this study was to assess the safety and efficacy of adalimumab in children with moderate-to-severe ulcerative colitis.
\end{abstract}

Methods The double-blind ENVISION I study was done at 24 hospitals in ten countries. Children (4-17 years) with moderate-to-severe ulcerative colitis despite stable doses of concurrent treatment with oral corticosteroids or immunosuppressants were enrolled. Per the original study design, patients were randomly assigned with an Interactive Voice Response System (IVRS) to receive either high-dose induction adalimumab $(2.4 \mathrm{mg} / \mathrm{kg}$ [maximum $160 \mathrm{mg}$ ] at weeks 0 and 1$)$ or standard-dose induction adalimumab $(2.4 \mathrm{mg} / \mathrm{kg}$ at week 0 and placebo at week 1); both groups received $1.2 \mathrm{mg} / \mathrm{kg}$ (maximum $80 \mathrm{mg}$ ) at week 2 and $0.6 \mathrm{mg} / \mathrm{kg}$ (maximum $40 \mathrm{mg}$ ) at weeks 4 and 6 . Patients with partial Mayo score (PMS) response at week 8 (defined as a decrease of two or more points and a decrease of $\geq 30 \%$ from baseline in PMS) were randomly assigned (2:2:1)—using IVRS—to receive either high-dose maintenance adalimumab $(0.6 \mathrm{mg} / \mathrm{kg}$ weekly), standard-dose maintenance adalimumab $(0.6 \mathrm{mg} / \mathrm{kg}$ every other week), or placebo up to week 52 (random assignment to the placebo group was ceased mid-trial, as was randomisation in the induction phase with all subsequent patients receiving open-label high-dose induction adalimumab). Coprimary endpoints were the proportion of patients with PMS remission at week 8 (intent-to-treat [ITT]-E population, not including those patients who were not randomised in the induction phase) and full Mayo score (FMS) remission at week 52 in week 8 PMS responders (maintenance ITT-E [mITT-E] population), for which the pooled adalimumab group (patients who received high-dose or standard-dose adalimumab) and the individual dose groups were compared against external adult placebo rates. We report results of the final confirmatory analysis. This trial is registered with ClinicalTrials.gov, NCT02065557.

Findings 93 children were recruited between Oct 13, 2014, and Sept 5, 2018, to the main study (77 [83\%] were randomly assigned [double-blind] to receive high-dose or standard-dose induction adalimumab; 16 [17\%] received open-label high-dose induction adalimumab after study design change). At week 8, $74(80 \%)$ children who were PMS responders continued to the maintenance period. $62(84 \%)$ patients were randomly assigned to receive high-dose or standarddose maintenance adalimumab treatment; $12(16 \%)$ patients received placebo. In patients in the ITT-E population who were randomly assigned to receive high-dose induction adalimumab, a significantly higher proportion of patients were in PMS remission at week 8 (28 [60\%] of 47) compared with external placebo $(19.8 \%$; $p=0.0001) .13(43 \%)$ of 30 patients in the standard-dose induction adalimumab group were in PMS remission at week 8 versus an external placebo rate of $19.8 \%$, but this difference was not significant $(\mathrm{p}=0 \cdot 38)$. Similarly, FMS remission at week 52 in children who were week 8 PMS responders was reported in a significantly higher proportion of patients in mITT-E population who received high-dose maintenance adalimumab (14 [45\%] of 31 patients) versus external placebo at week $52(18.4 \% ; \mathrm{p}=0 \cdot 0001)$. Nine $(29 \%)$ of 31 patients in the standard-dose maintenance adalimumab group were in FMS remission at week 52 versus an external placebo rate of $18 \cdot 4 \%$, but this difference was not significant $(\mathrm{p}=0 \cdot 38)$. Remission rates in the pooled adalimumab groups were significantly better compared with external placebo (PMS remission at week 8: 41 [53\%] of 77 patients; $p<0 \cdot 0001$; FMS remission at week $52: 23$ [37\%] of 62 patients; $p=0 \cdot 0001$ ). $21(23 \%)$ of 93 patients in the main study had one or more treatment-emergent serious adverse events during any adalimumab exposure. The most common adverse events were headache, anaemia, and ulcerative colitis flare during the induction period and ulcerative colitis flare, headache, and nasopharyngitis during the maintenance period.

Interpretation Clinically meaningful rates of remission and response were reported in children who received adalimumab in this study. No new safety signals were observed, suggesting that adalimumab is an efficacious and safe treatment option for children with moderate-to-severe ulcerative colitis.

Funding AbbVie.
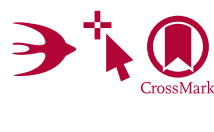

Lancet Gastroenterol Hepatol 2021

Published Online

June 18,2021

https://doi.org/10.1016/

S2468-1253(21)00142-4

See Online/Comment

https://doi.org/10.1016/

S2468-1253(21)00173-4

Centre for Immunobiology, Blizard Institute, Barts and the London School of Medicine, Queen Mary University of London, London, UK (Prof N M Croft MBBS); Department of Paediatric Gastroenterology, The Royal London Children's Hospital, Barts Health NHS Trust, London, UK (Prof N M Croft); Gastroenterology and Hepatology, Mayo Clinic, Rochester, MN, USA (W A Faubion Jr MD); School of Medicine, Emory University, Atlanta, GA, USA

(Prof S Kugathasan MD); Children's Health Care of Atlanta, Atlanta, GA, USA (Prof S Kugathasan); The Children's Memorial Health Institute, Warsaw, Poland (ProfJ Kierkus MD); Université de Paris, APHP-Hôpital Necke Enfants Malades, Service de Gastroentérologie pédiatrique, Paris, France

(Prof F M Ruemmele MD); Department of Pediatrics and Adolescent Medicine, Graduate School of Medicine, Juntendo University, Tokyo, Japan (Prof T Shimizu MD); AbbVie, North Chicago, IL, USA (N M Mostafa PhD, $M$ Venetucci BS,

T Finney-Hayward PhD, Y Sanchez Gonzalez PhD); AbbVie Deutschland, Ludwigshafen, Germany (M Bereswill MS, A Lazar MD); Shaare Zedek Medical Center, Hebrew University of Jerusalem, Jerusalem, Israel (Prof D Turner MD) 
Correspondence to: Prof Nicholas M Croft, Centre for Immunobiology, Blizard Institute, Barts and the London School of Medicine, Queen Mary University of London, London E1 2AT, UK n.m.croft@qmul.ac.uk
Research in context

\section{Evidence before this study}

Before the initiation of the present study in 2014, clinical trials in paediatric patients with moderate-to-severe ulcerative colitis were scarce. The only relevant studies were an open-label study of 60 children with moderate-to-severe ulcerative colitis that evaluated efficacy and safety of the tumour necrosis factor (TNF) inhibitor infliximab through 54 weeks, and a multicentre registry study of 52 children receiving infliximab that evaluated clinical activity, colectomy rate, and serious adverse events. We searched PubMed for articles published from Jan 1, 2014, to Dec 22, 2020, using search terms "ulcerative colitis (UC)" "pediatric/children" "tumor necrosis factor (TNF)" and "biologic." Of the 192 articles retrieved, we identified only one additional openlabel clinical study that investigated the use of TNF inhibitor (golimumab) in children with moderate-to-severe ulcerative colitis and 11 real-world or registry studies that reported data for biologic use in paediatric patients with inflammatory bowel disease, including ulcerative colitis. None of the studies included an internal placebo group, and most of the studies were small (4-76 patients with ulcerative colitis) and reported limited efficacy and safety data.

\section{Added value of this study} ENVISION I is the largest randomised, double-blind, phase 3 study of a biologic therapy in paediatric patients with ulcerative colitis done to date. The findings of the study show that adalimumab treatment was efficacious and well tolerated in children with moderate-to-severe ulcerative colitis with clinically meaningful rates of remission and response, including corticosteroid-free remission and mucosal healing, observed up to 52 weeks.

\section{Implications of all the available evidence}

On the basis of the available evidence, TNF inhibitors, such as adalimumab, appear to be well tolerated and efficacious for the treatment of children with moderate-to-severe ulcerative colitis.

\section{Introduction}

Ulcerative colitis is a chronic inflammatory bowel disease that affects children and adults. ${ }^{1-3}$ Previous studies have reported that $8-25 \%$ of patients present with symptoms before the age of 18 years, and the incidence is increasing in children. ${ }^{47}$ The disease course tends to be more severe in the paediatric population ( $<18$ years old), with $82 \%$ of paediatric-onset cases having extensive involvement compared with $47 \%$ of adult-onset cases. Additionally, paediatric-onset cases are associated with a higher need for immunosuppressants..$^{1-3}$

Additional age-related considerations exist when treating children with ulcerative colitis-including maintenance of growth potential, nutrition, and bone mineral density - all of which are important in growing children and some of which can be negatively affected by prolonged corticosteroid use. ${ }^{1,8,9}$ Other important considerations when treating children with ulcerative colitis include dissatisfaction with body image, self-esteem issues, and social interactions..$^{10,11}$

The chimeric tumour necrosis factor (TNF) inhibitor infliximab was the only biologic therapy approved for paediatric ulcerative colitis before the approval of adalimumab. Infliximab requires infusions given in hospital or at home by health-care professionals. Because of its immunogenicity, it is often given with an immunomodulator to reduce the risk of loss of response..$^{12-15}$ Thus, an unmet therapeutic need exists.

Adalimumab is a subcutaneously and in-home administered, fully human, monoclonal TNF inhibitor approved for adult and-as of September, 2020 paediatric, moderate-to-severe ulcerative colitis, adult and paediatric Crohn's disease, and other immunemediated inflammatory diseases. ${ }^{16}$ The objective of the phase 3 ENVISION I study was to assess efficacy by showing the superiority of adalimumab over external placebo and the safety of adalimumab in children with moderate-to-severe ulcerative colitis.

\section{Methods}

\section{Study design and participants}

ENVISION I was designed as a double-blind, placebocontrolled phase 3 trial. After a change to the study design, an external placebo comparator was used. The study included an 8-week induction period followed by a 44-week maintenance period. The study enrolled children (aged 4-17 years) with moderate-to-severe ulcerative colitis at 19 sites in Austria, Belgium, Canada, Spain, the UK, Israel, Poland, Slovakia, and the USA (appendix p 2). In addition, a substudy was done at five sites in Japan to satisfy local regulatory requirements. To ensure a fully comprehensive report, the integrated data of the main study and the Japanese substudy are included in the appendix. Unless otherwise noted, results presented within this manuscript are based on main study data (excluding the Japanese patients).

Eligible patients were children (4-17 years) diagnosed with ulcerative colitis 12 weeks or more before screening, who had active, moderate-to-severe ulcerative colitis (defined as full Mayo score [FMS] of 6-12 and endoscopy subscore of $2-3$, confirmed by a central reader) despite stable doses of concurrent treatment with oral corticosteroids $(\geq 2 \mathrm{mg}$ per day oral prednisone or equivalent, not exceeding $40 \mathrm{mg}$ per day, or $\geq 3 \mathrm{mg}$ per day oral budesonide, not exceeding $9 \mathrm{mg}$ per day) or immunosuppressants (methotrexate $\geq 15 \mathrm{mg} / \mathrm{m}^{2}$ body surface area per week, azathioprine $\geq 1.5 \mathrm{mg} / \mathrm{kg}$ per day, or mercaptopurine $\geq 1 \mathrm{mg} / \mathrm{kg}$ per day). Patients on both oral corticosteroids and immunosuppressants were required to fulfil the criteria for both drugs. Patients who had 
documented intolerance or no response to corticosteroids or immunosuppressants were also eligible. Patients also needed to have tested negative for tuberculosis at screening assessment.

Patients were excluded if they had Crohn's disease, unclassified inflammatory bowel disease, fulminant colitis, toxic megacolon, ulcerative colitis disease restricted to the rectum, history of colectomy, or planned bowel surgery; previous adalimumab treatment; and those receiving ciclosporin, tacrolimus, or mycophenolate mofetil within 30 days before baseline, infliximab within 56 days of baseline, or therapeutic enema or suppository within 14 days before the screening endoscopy. Furthermore, unless the patient had a treatment limiting reaction, patients who had previously used infliximab or any anti-TNF drug and had not clinically responded at any time were excluded. Patients who had positive results for hepatitis B surface antigen or DNA were excluded, as were patients with evidence of dysplasia or history of malignancy other than successfully treated non-metastatic cutaneous squamous cell or basal cell carcinoma or localised carcinoma in situ of the cervix. Patients with clinically significant abnormalities on screening electrocardiogram, aspartate aminotransferase or alanine aminotransferase concentrations more than 1.75-times the upper limit of normal, total bilirubin concentration of $3 \mathrm{mg} / \mathrm{dL}$ or more, or serum creatinine concentrations of more than $1.6 \mathrm{mg} / \mathrm{dL}$ were also excluded.

Patients taking concomitant immunosuppressants needed to remain stable for the first 12 weeks of the study (except in the event of treatment-related toxicities) and could discontinue therapy starting at week 12 per investigator's discretion. Patients who were receiving concomitant medications and who had disease flare at or after week 12 were allowed to change their dose or initiate treatment with corticosteroids, immunosuppressants, or aminosalicylates if the dose increase was discussed with and approved by the medical monitor. Patients taking concomitant corticosteroids were not allowed to change dose during the first 4 weeks of the study but could start a corticosteroid taper at or after week 4 at investigator's discretion.

The inclusion and exclusion criteria were the same in the Japanese substudy, with the exception of the doses of concurrent azathioprine $(\geq 1.0 \mathrm{mg} / \mathrm{kg}$ per day) or 6-mercaptopurine ( $\geq 0.6 \mathrm{mg} / \mathrm{kg}$ per day), and additional exclusion criteria: cytapheresis treatment within 56 days before baseline or antibiotic combination therapy (amoxicillin, tetracycline, and metronidazole) during the screening period.

The study was done in accordance with International Council for Harmonisation guidelines and the ethical principles of the Declaration of Helsinki. All patients provided written informed consent, and the study protocol was approved by an institutional review board or independent ethics committee at each study site.

\section{Randomisation and masking}

Patients were assigned a unique identification number by the Interactive Voice Response System (IVRS) or Interactive Web Response System (IWRS) when screened for the study. Patients were centrally randomly assigned to a treatment group with IVRS or IWRS according to the randomisation scheme generated by the study sponsor (AbbVie, North Chicago, IL, USA). Treatment group assignment was maintained by the IVRS and not provided to the site. The sites were provided with appropriate kit numbers for drug-dispensing purposes for each patient by the IVRS. Under the original study design, at the beginning of the 8-week double-blind induction period, eligible patients were randomly assigned (3:2) to receive either high-dose or standard-dose induction adalimumab. The 3:2 randomisation at baseline was stratified by baseline disease severity (FMS $\leq 9$ vs $>9$ ), baseline corticosteroid use (yes vs no), and previous exposure to TNF inhibitors (yes vs no) using blocks of five.

After the 8-week induction period, patients who were in partial Mayo score (PMS) response (ie, were PMS responders) at week 8 (defined as a decrease of two points or more and $\geq 30 \%$ decrease from baseline PMS) were eligible to enter the 44-week, double-blind maintenance period. In the original study design, patients were randomly assigned $(2: 2: 1)$ at week 8 to receive high-dose maintenance adalimumab, standard-dose maintenance adalimumab, or placebo. Non-responders at week 8 were discontinued from the study (appendix p 10). The 2:2:1 rerandomisation at week 8 was stratified by week 8 PMS remission status (yes vs no) and induction dose (high-dose vs standard-dose induction adalimumab) using blocks of five (appendix pp 10-11).

A study design change was introduced in November, 2017, that ceased random assignment to the maintenance placebo group at week 8 (appendix p 11). The cessation of placebo was due to slow recruitment because of inadequate acceptance of placebo by both families and investigators. This change was agreed to by the regulatory agencies. In addition, random assignment into the standard-dose induction adalimumab group at baseline was terminated to increase the chance of randomly assigning responders to maintenance therapy based on the original premise of the higher efficacy of high-dose versus standard-dose adalimumab (protocol amendment 4; Nov 2, 2017; incorporated in amendment 5; appendix pp 47-49 and pp 117-118). Thus, all patients enrolled in the study after the study design change received open-label high-dose induction adalimumab. The maintenance period remained double-blind. All week 8 PMS responders (including patients who had been enrolled in the study before the study design change and had not yet entered the maintenance period) were randomly assigned (1:1) to receive either high-dose maintenance adalimumab or standard-dose maintenance adalimumab. Randomisation at week 8 was stratified by week 8 PMS remission status (yes vs no) using a block of two. 
At or after week 12, patients with a disease flare could be randomly assigned to receive one-time, double-blind reinduction treatment with adalimumab or a one-time, double-blind regular maintenance dose of adalimumab at the visit. All patients continued their original randomly assigned high-dose or standard-dose maintenance adalimumab thereafter, except patients in the placebo group, who continued on standard-dose maintenance adalimumab.

The investigators, study site personnel, patients, and all AbbVie personnel with direct oversight of the conduct and management of the trial (with the exception of the AbbVie Drug Supply Management Team) remained masked to each patient's treatment throughout the double-blind periods of the study.

\section{Procedures}

During the 8-week induction period, eligible patients received either high-dose $(2.4 \mathrm{mg} / \mathrm{kg}$ [maximum $160 \mathrm{mg}$ ] at weeks 0 and 1$)$ or standard-dose $(2.4 \mathrm{mg} / \mathrm{kg}$ [maximum $160 \mathrm{mg}$ ] at week 0 and matching placebo at week 1) induction adalimumab subcutaneously. Both groups received $1.2 \mathrm{mg} / \mathrm{kg}$ (maximum $80 \mathrm{mg}$ ) adalimumab at week 2 and $0.6 \mathrm{mg} / \mathrm{kg}$ (maximum $40 \mathrm{mg}$ ) adalimumab at weeks 4 and 6 (appendix p 10). During the maintenance period, patients received either high-dose maintenance adalimumab $(0.6 \mathrm{mg} / \mathrm{kg}$ weekly [maximum $40 \mathrm{mg}$ ]) or standard-dose maintenance adalimumab $(0.6 \mathrm{mg} / \mathrm{kg}$ every other week [maximum $40 \mathrm{mg}$ ]).

Following a flare, patients were randomly reassigned to receive either reinduction treatment with adalimumab at a dose of $2.4 \mathrm{mg} / \mathrm{kg}$ (maximum $160 \mathrm{mg}$ ) or a maintenance dose of $0.6 \mathrm{mg} / \mathrm{kg}$ (maximum $40 \mathrm{mg}$ ). If the disease flare was still present after 4 weeks, patients could be switched to open-label adalimumab $0.6 \mathrm{mg} / \mathrm{kg}$ (maximum $40 \mathrm{mg}$ ) weekly, and if the flare persisted for an additional 4 weeks, the patients could switch to open-label adalimumab $40 \mathrm{mg}$ weekly (not weight based). Thereafter, patients with persistent disease flare could be withdrawn from the study at the investigator's discretion. During openlabel rescue therapy, patients who were responders and had been in remission for 8 consecutive weeks or more (PMS $\leq 2$ with no individual subscore $>1$ ) could have their dosage decreased from every week to every other week. Flare was defined as patients with a week 8 PMS of 0-2 that increased by three points or more, patients with a week 8 PMS of 3 or 4 that increased by two points or more, and patients with a week 8 PMS of 5 or 6 that increased by one point or more.

A patient could withdraw from the study at any time, and the investigator could withdraw a patient for any reason. Dose interruptions were permitted per protocol if a patient developed an infection requiring intravenous anti-infective treatment, if an infection met the definition of serious, or 2 weeks before an elective surgery or at the time of an emergency surgery. Study drug could be reinstated per physician's judgement.
Endoscopies were done at screening and at week 52 or at premature discontinuation. All endoscopies were reviewed by a central reviewer who was masked to the therapy and time point.

Blood samples were obtained at weeks 0, 2, 4, 8, 12, 26, 34, and 52 for pharmacokinetic evaluation of serum adalimumab concentrations and at weeks $0,4,8,26$, and 52 for evaluation of anti-adalimumab antibodies by a validated enzyme-linked immunosorbent assay based on a double-antigen technique. Adverse events and laboratory data were assessed throughout the study.

\section{Outcomes}

The coprimary efficacy endpoints were the proportion of patients who were in PMS remission at week 8 (intent-to-treat [ITT]-E population) and the proportion of week 8 PMS responders who were in FMS remission, defined as FMS of two or less and no individual subscore more than one, at week 52 (maintenance ITT-E [mITT-E] population).

The four ranked secondary endpoints at week 52 were the proportion of patients who showed FMS clinical response (defined as a decrease of three points or more and $\geq 30 \%$ decrease from baseline in FMS) in week 8 PMS responders, mucosal healing (defined as Mayo endoscopy subscore of one or less by central reading) in week 8 PMS responders, FMS remission in week 8 PMS remitters, and corticosteroid-free FMS remission in week 8 PMS responders who were taking corticosteroids at baseline (mITT-E population).

Other secondary endpoints included change from baseline in total IMPACT III Quality of Life score and subscores for patients 9 years or older at baseline; change in stool frequency and rectal bleeding Mayo subscores; change in $\mathrm{z}$ scores for height velocity and body mass index (BMI) at weeks 26 and 52; and change in Work Productivity and Activity Impairment (WPAI) questionnaire caregiver scores for absenteeism, presenteeism, work impairment, and activity impairment. In addition, the proportion of week 8 PMS responders in Pediatric Ulcerative Colitis Activity Index (PUCAI) remission (defined as PUCAI $<10$ ) or PUCAI response (defined as a decrease in PUCAI $\geq 20$ points from baseline) was assessed at weeks 8 and 52 . Week 8 endpoints were assessed in the ITT-E population, week 52 endpoints and change from baseline assessments were assessed in the mITT-E population. A full list of secondary outcomes is reported in the appendix (pp 104-106).

Safety was assessed by recording adverse events and laboratory values. Treatment-emergent adverse events were defined as events with an onset date after the first dose of study medication and not more than 70 days after the last dose of study medication.

\section{Statistical analysis}

Several analysis populations were included in this study (appendix p 4). The ITT and safety population included 


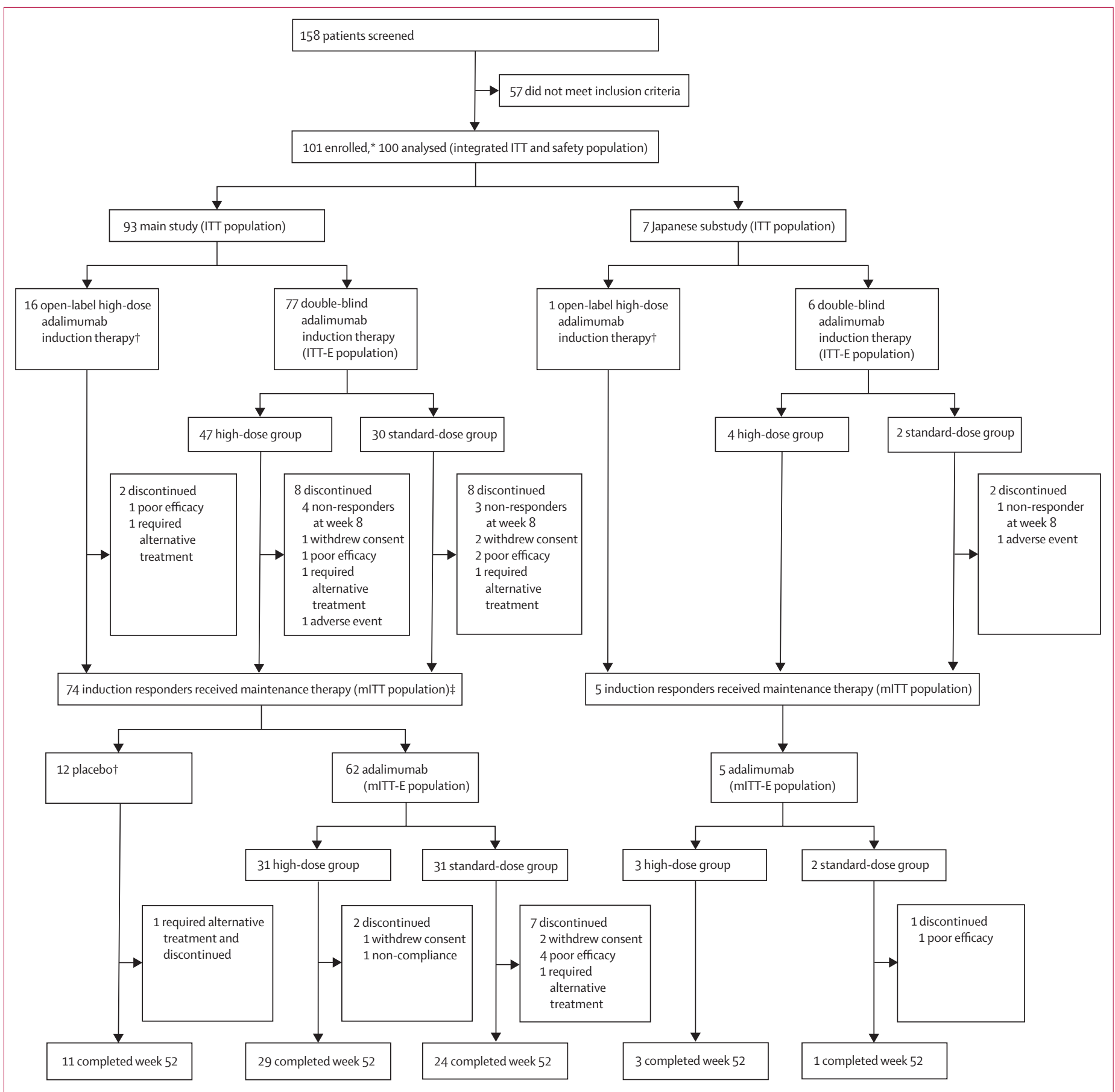

Figure 1: Trial profile

ITT and safety population included all patients who received at least one induction dose of adalimumab (double-blind or open-label). ITT-E population included patients enrolled before study design change who received double-blind induction adalimumab. $\mathrm{mITT}$ population included all week 8 responders randomly assigned to receive a maintenance regimen who received at least one dose of maintenance adalimumab. $\mathrm{mITT}$ - E population included week 8 responders who received high-dose or standard-dose maintenance adalimumab. ITT=intent to treat. $\mathrm{mITT}=$ maintenance ITT.

PMS=partial Mayo score. *A Japanese patient's treatment in the study was still ongoing at the time of this final confirmatory analysis; this patient was excluded from all analyses. $\dagger$ After the study design change, patients received open-label high-dose adalimumab only during the induction period and random assignment to the placebo group was ceased. ‡One additional non-responder who was erroneously randomly assigned into the maintenance period was excluded from the mITT-E population efficacy analyses, but was analysed as part of the safety population. 
all patients who received one or more doses of the study drug. The ITT-E population, which was the primary population for the week 8 confirmatory efficacy analysis, was defined as all patients who received one or more doses of the study drug during the induction period randomly assigned before the study design change (protocol amendment 4). This was done to exclude patients allocated to the open-label, high-dose induction adalimumab group to avoid introducing any bias. The mITT-E population, which was the primary population for the week 52 confirmatory efficacy analysis, was defined as all week 8 PMS responders who were randomly assigned at week 8 to the maintenance period and received one or more doses of high-dose or standarddose maintenance adalimumab; patients who were randomly assigned to receive placebo were excluded. The Japanese substudy used the same population definitions. The modifier 'integrated' indicates that the analysis population contained patients from the main and Japanese substudy.

Pharmacokinetic and immunogenicity assessments were done in the integrated analysis populations, and

\begin{tabular}{|c|c|c|c|}
\hline & $\begin{array}{l}\text { ITT and safety } \\
\text { population* }(n=93)\end{array}$ & $\begin{array}{l}\text { ITT-E population } \dagger \\
(n=77)\end{array}$ & $\begin{array}{l}\text { mITT-E population } \ddagger \\
(n=62)\end{array}$ \\
\hline Girls & $51(55 \%)$ & $38(49 \%)$ & $36(58 \%)$ \\
\hline Boys & $42(45 \%)$ & $39(51 \%)$ & $26(42 \%)$ \\
\hline \multicolumn{4}{|l|}{ Age, years } \\
\hline Mean age & $14 \cdot 1(3 \cdot 0)$ & $14 \cdot 1(3 \cdot 0)$ & $13 \cdot 7(3 \cdot 3)$ \\
\hline Median age & $15(12-17)$ & $15(12-17)$ & $14(11-17)$ \\
\hline Participants $<13$ years & $25(27 \%)$ & $20(26 \%)$ & $20(32 \%)$ \\
\hline \multicolumn{4}{|l|}{ Ethnicity } \\
\hline White & $88(95 \%)$ & $73(95 \%)$ & $59(95 \%)$ \\
\hline Black & $3(3 \%)$ & $3(4 \%)$ & $3(5 \%)$ \\
\hline Asian & $1(1 \%)$ & $1(1 \%)$ & 0 \\
\hline Mixed race & $1(1 \%)$ & 0 & 0 \\
\hline \multicolumn{4}{|l|}{ Geographic region } \\
\hline Eastern Europe & $72(77 \%)$ & $60(78 \%)$ & $49(79 \%)$ \\
\hline North America & $13(14 \%)$ & $10(13 \%)$ & $10(16 \%)$ \\
\hline Western Europe & $8(9 \%)$ & $7(9 \%)$ & $3(5 \%)$ \\
\hline Mean weight, kg & $55 \cdot 9(18 \cdot 1)$ & $56.7(18.1)$ & $55 \cdot 5(19 \cdot 6)$ \\
\hline Pancolitis & $56(60 \%)$ & $45(58 \%)$ & $37(60 \%)$ \\
\hline Anti-TNF naive & $78(84 \%)$ & $63(82 \%)$ & $54(87 \%)$ \\
\hline Baseline systemic corticosteroid use & $44(47 \%)$ & $35(45 \%)$ & $29(47 \%)$ \\
\hline Baseline immunosuppressant use & $55(59 \%)$ & $50(65 \%)$ & $38(61 \%)$ \\
\hline \multicolumn{4}{|l|}{ Duration of ulcerative colitis, years } \\
\hline Mean duration & $2 \cdot 3(2 \cdot 3)$ & $2 \cdot 3(2 \cdot 3)$ & $2 \cdot 2(2 \cdot 0)$ \\
\hline Median duration & $1 \cdot 5(0 \cdot 8-2 \cdot 6)$ & $1 \cdot 5(0.9-2 \cdot 8)$ & $1.5(0.9-2 \cdot 9)$ \\
\hline Mean full Mayo score & $7 \cdot 8(1 \cdot 2)$ & $7 \cdot 8(1 \cdot 2)$ & $7 \cdot 8(1 \cdot 2)$ \\
\hline Mean partial Mayo score & $5 \cdot 6(1 \cdot 2)$ & $5 \cdot 7(1 \cdot 2)$ & $5.7(1.1)$ \\
\hline \multicolumn{4}{|c|}{$\begin{array}{l}\text { Data are } n(\%) \text {, mean }(S D) \text {, or median (IQR). ITT=intent-to-treat. mITT-E=maintenance ITT-E. TNF=tumour necrosis } \\
\text { factor. *ITT and safety population included patients receiving one or more induction dose of adalimumab (double- } \\
\text { blind or open-label). IITT-E population included patients enrolled before design change who received double-blind } \\
\text { induction adalimumab. fmITT-E population included week } 8 \text { responders randomly assigned to maintenance period } \\
\text { receiving high-dose or standard-dose maintenance adalimumab. }\end{array}$} \\
\hline
\end{tabular}

patients were categorised based on their dose (ie, the high-dose induction adalimumab group included masked and open-label treatment).

Following the study design change, in the confirmatory efficacy analysis, coprimary and ranked secondary endpoints were tested for the adalimumab groups against historical, external adult placebo rates derived from a meta-analysis of suitable clinical studies of similar study design, patient populations, and endpoint definitions (ULTRA $1^{17}$ and ULTRA $2^{18}$ for week 8 coprimary endpoint; GEMINI $1^{19}$ and OCTAVE sustain ${ }^{20}$ for week 52 coprimary and ranked secondary endpoints). A sequentially rejective multiple test procedure controlled the multiple significance level of $5 \%$ using one-sample, two-sided $\chi^{2}$ tests. Coprimary and ranked secondary endpoints were tested for in the pooled adalimumab group versus external placebo first, followed by testing of individual dose groups versus external placebo separately. Adjusted $\mathrm{p}$ values from the sequentially rejective multiple test procedure are presented (ie, statistical significance if the corresponding adjusted $\mathrm{p}$ value was less than $0 \cdot 05$ ).

An underlying sample size calculation showed that, for the week 8 coprimary endpoint, 77 patients in the ITT-E population (46 [60\%] in the high-dose induction adalimumab group) would provide at least $99 \%$ power for a one-sample, two-sided $\chi^{2}$ test with a significance level of $5 \%$ to compare the pooled induction adalimumab group (assuming a 48\% PMS remission rate at week 8) with an assumed $19.8 \%$ external placebo PMS remission rate at week 8 and at least $99 \%$ power with a significance level of $4.95 \%$ to compare high-dose induction adalimumab group (assuming a 52\% PMS remission rate at week 8) with an assumed $19.8 \%$ external placebo PMS remission rate at week 8 . For the week 52 coprimary endpoint, 57 patients in the mITT-E population (28 [49\%] in the high-dose maintenance adalimumab group) would provide $88 \%$ power for a one-sample, two-sided $\chi^{2}$ test with a significance level of $5 \%$ to compare the pooled maintenance adalimumab group (assuming a 36\% FMS remission rate at week 52 ) with an assumed $18.4 \%$ external placebo FMS remission rate at week 52 and at least $80 \%$ power with a significance level of $5 \%$ to compare high-dose maintenance adalimumab group (assuming a 41\% FMS remission rate at week 52) with an assumed 18. 4\% external placebo FMS remission rate at week 52 .

Binary endpoints were summarised as proportions by treatment group, including 95\% CIs. Continuous endpoints were summarised as changes from baseline and reported by treatment group, including 95\% CIs.

Missing values were imputed using non-responder imputation for binary efficacy variables and last observation carried forward for categorical and continuous variables. Missing values were only imputed for study periods (before week 8 and after week 8 ) that a patient had actually entered. Subgroup analyses of coprimary endpoints were done by age, weight, previous TNF 


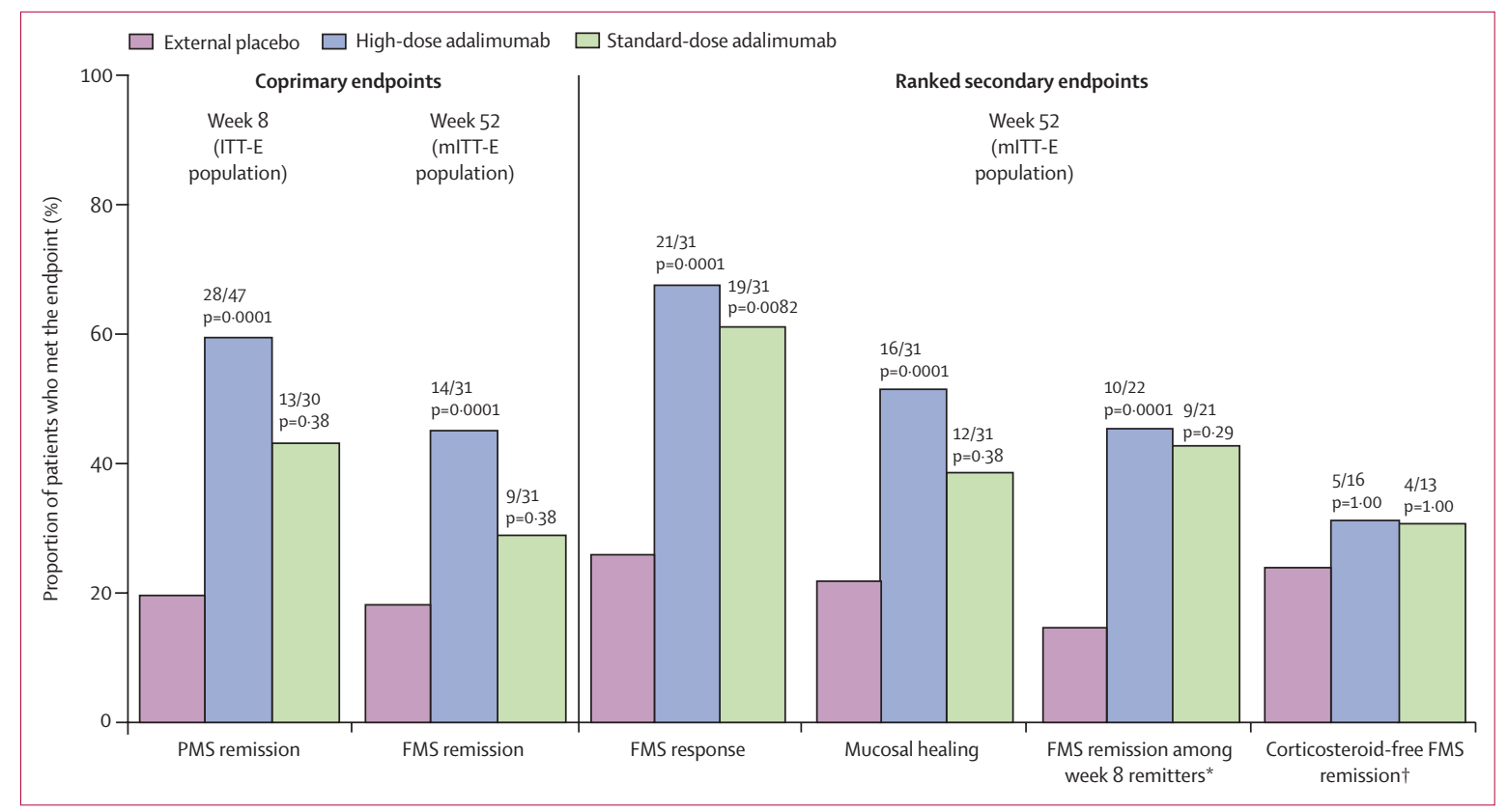

Figure 2: Coprimary and ranked secondary endpoints

Analysis populations were ITT-E for week 8 coprimary endpoint and mITT-E for week 52 coprimary and secondary endpoints. Adjusted $p$ values from the prespecified sequentially rejective multiple test procedure (controlling multiple significance level of $5 \%$ ) using one-sample two-sided $\chi^{2}$ tests for the high-dose and standard-dose adalimumab groups versus external placebo are reported. Week 52 analyses were done in week 8 PMS responders unless otherwise noted. FMS=full Mayo score. ITT-E=intent to treat. mITT-E=maintenance ITT. PMS=partial Mayo score. *Analysis in week 8 remitters. $†$ Analysis in patients with baseline corticosteroid use.

inhibitor therapy, baseline corticosteroid or immunosuppressant use, and geographical region. Adverse events were analysed separately for the induction period, maintenance period, after disease flare, and during overall adalimumab exposure.

We report the final confirmatory analysis. The statistical analyses were done using the SAS (version 9.4). This study is registered with ClinicalTrials.gov, NCT02065557.

\section{Role of the funding source}

AbbVie funded this study and participated in the study design, research, analysis, data collection, interpretation of data, reviewing, and approval of the publication. No honoraria or payments were made for authorship.

\section{Results}

In the main study, 93 children were recruited between Oct 13, 2014, and Sept 5, 2018. 77 (83\%) of 93 patients received double-blind adalimumab (47 [61\%] in the high-dose induction adalimumab group and 30 [39\%] in the standard-dose induction adalimumab group) during the 8-week induction period (ITT-E population), and $16(17 \%)$ of 93 patients received open-label high-dose induction adalimumab (figure 1). At week 8, 77 (83\%) patients were PMS responders; 74 (80\%) responders continued to the maintenance period (figure 1): 62 (84\%) were randomly assigned to receive adalimumab (pooled maintenance adalimumab group; 31 [50\%] in the highdose maintenance adalimumab group and $31[50 \%]$ in the standard-dose maintenance adalimumab group; mITT-E population), and $12(16 \%)$ were randomly assigned to receive placebo (before the study design change). A total of 22 patients were randomly reassigned due to disease flare during the maintenance period: 15 (68\%) in the pooled maintenance adalimumab group (nine [60\%] in the high-dose maintenance adalimumab group [three with reinduction and six without reinduction]; and six [40\%] in the standard-dose maintenance adalimumab group [three with reinduction and three without reinduction]) and seven (32\%) in the placebo group.

Median exposure to study drug was 56 days (IQR 55-56) for the 93 patients in the induction period; 307 days (97-308) for 75 (81\%) patients during the maintenance period up to first flare, if applicable; and 161 days (84-257) for the 22 (29\%) patients after flare. Mean age was $14 \cdot 1$ (SD 3.0) years, 51 (55\%) of the 93 participants were girls, and median duration of ulcerative colitis was $1 \cdot 5$ years (IQR $0 \cdot 8-2 \cdot 6$ ) in the ITT and safety population (table 1). At baseline, 44 (47\%) of 93 patients used systemic corticosteroids, 55 (59\%) patients used immunosuppressants, and 78 (84\%) patients were TNF inhibitor naive.

Eight additional patients were enrolled in the Japanese substudy until March 4, 2019; however, at the time of confirmatory analysis of the study, one patient's treatment was still ongoing. Thus, only seven Japanese patients were included in the integrated ITT and safety population ( $\mathrm{n}=100$; figure 1$)$. 


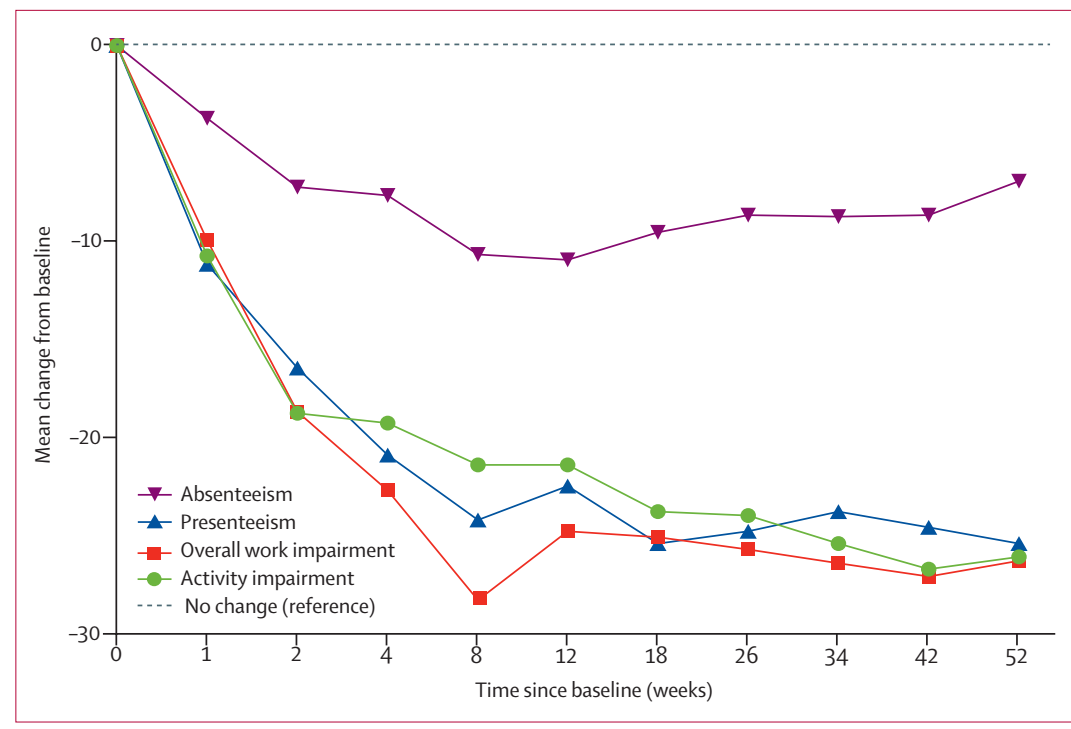

Figure 3: Mean change from baseline in caregivers' WPAI scores in the mITT-E population (LOCF) $\mathrm{LOCF}=$ last observation carried forward. $\mathrm{mITT}=$ maintenance intent to treat. WPAI=Work Productivity and Activity Impairment questionnaire. Results from the pooled maintenance adalimumab group are presented.

In the integrated analysis population, median (IQR) exposure to study drug was 56 days (IQR 55-56) for 100 patients during the induction period; 307 days (94-308) for $80(80 \%)$ patients in the maintenance period up to first flare, if applicable; and 162 days (94-245) for $25(31 \%)$ patients after flare. Baseline characteristics were similar in the integrated ITT and integrated safety population (appendix p 5).

In the ITT-E population, the pooled induction adalimumab group was superior to external placebo for the coprimary endpoint of PMS remission at week 8 (41 [53\%] of 77 patients vs $19.8 \%$ for external placebo; adjusted $\mathrm{p}<0 \cdot 0001)$. Similarly, in the mITT-E population, the pooled maintenance adalimumab group was superior to external placebo for the coprimary endpoint of FMS remission at week 52 (23 [37\%] of 62 patients vs $18.4 \%$ for external placebo; adjusted $\mathrm{p}=0 \cdot 0001)$.

A significantly higher proportion of patients in the ITT-E population who received high-dose induction adalimumab (28 [60\%] of 47 patients) were in PMS remission at week 8 versus external placebo $(19.8 \%$; adjusted $\mathrm{p}=0 \cdot 0001) ; 13$ (43\%) of 30 patients who received standard-dose induction adalimumab were in PMS remission at week 8 versus an external placebo rate of $19.8 \%$, but this difference was not significant $(\mathrm{p}=0 \cdot 38$; figure 2).

A significantly higher proportion of patients in the mITT-E population who received high-dose maintenance adalimumab were in FMS remission at week 52 (14 [45\%] of 31 patients) compared with external placebo $(18 \cdot 4 \%$; adjusted $\mathrm{p}=0 \cdot 0001)$; nine $(29 \%)$ of 31 patients who received standard-dose maintenance adalimumab were in FMS remission at week 52 versus an external placebo rate of $18.4 \%$, but this difference was not significant

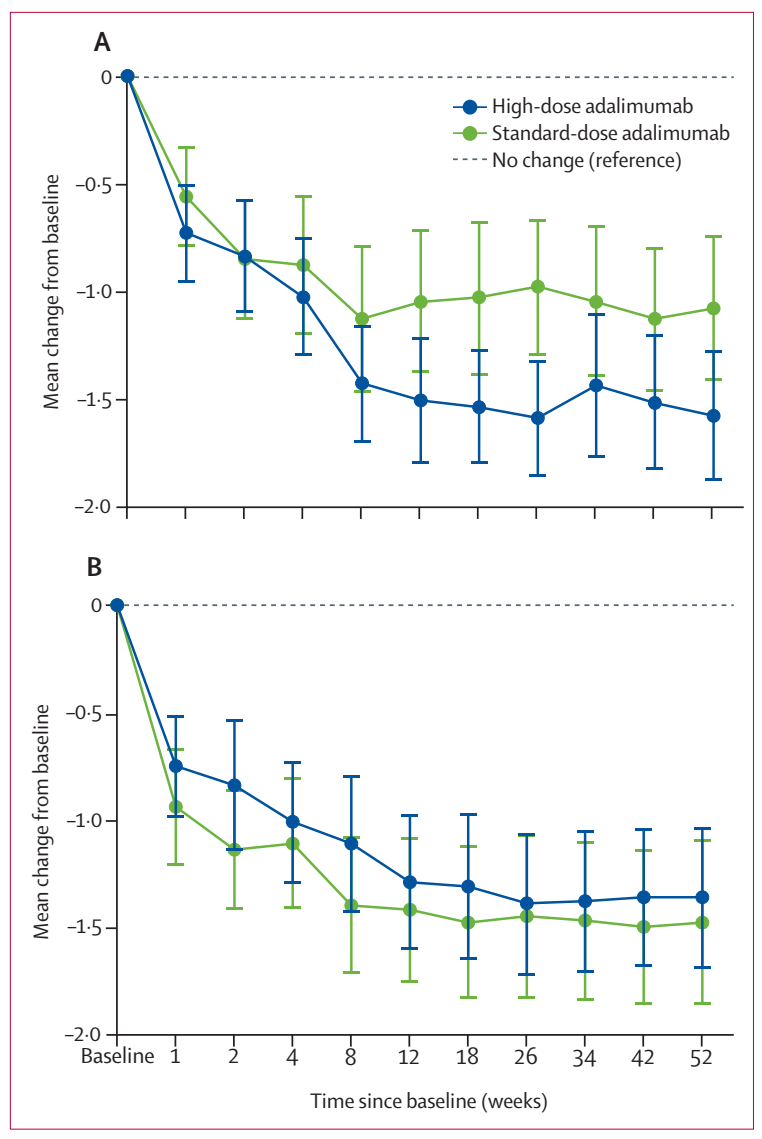

Figure 4: Mean change from baseline in stool frequency $(A)$ and rectal bleeding (B) Mayo subscores in the mITT-E population (LOCF)

Error bars are $95 \% \mathrm{Cl}$. LOCF=last observation carried forward. $\mathrm{mITT}=$ maintenance intent to treat.

$(\mathrm{p}=0 \cdot 38$; figure 2$)$. Of note, none of the patients who were in FMS remission at week 52 were receiving steroids (either never during the study or had discontinued use before week 52).

In the mITT-E population, compared with external placebo, a significantly higher proportion of patients who received high-dose maintenance adalimumab reported FMS response in week 8 PMS responders (21 [68\%] of 31 patients vs $26 \cdot 1 \%$; $\mathrm{p}=0 \cdot 0001)$, mucosal healing in week 8 PMS responders (16 [52\%] of 31 patients vs $22 \cdot 0 \% ; \mathrm{p}=0 \cdot 0001)$, and FMS remission in week 8 remitters (10 [45\%] of 22 patients vs $14 \cdot 8 \%$; $\mathrm{p}=0 \cdot 0001)$ at week 52 (figure 2). Similarly, the pooled maintenance adalimumab group showed superiority over external placebo for these endpoints (all adjusted $\mathrm{p}=0 \cdot 0001$; data not shown). Of the patients in the mITT-E population who received standard-dose maintenance adalimumab, the proportion of patients with FMS response at week 52 was significantly higher compared with external placebo (19 [61\%] of 31 patients vs $26 \cdot 1 \%$; $\mathrm{p}=0 \cdot 0082$ ); there was no significant difference compared with external placebo for the three other secondary endpoints (figure 2). Mucosal healing was reported in 12 (39\%) of 31 patients 
in the standard-dose maintenance adalimumab group at week 52.

Although more patients who received high-dose adalimumab reported PMS remission at week 8 and FMS remission, FMS response, mucosal healing at week 52 in week 8 PMS responders, and FMS remission at week 52 in week 8 remitters than those who received standard-dose adalimumab (figure 2), the study was not powered for a formal statistical comparison between the two adalimumab dose regimens, and this was an exploratory analysis only.

Results were similar in the integrated analysis populations for both coprimary endpoints and ranked secondary endpoints (appendix $p$ 12); the results in Japanese patients were consistent with the overall integrated analysis populations.

In the ITT-E population, at the end of the induction period at week 8 clinical remission per PUCAI was reported in 22 (47\%) of 47 patients who received high-dose induction adalimumab and ten $(33 \%)$ of 30 patients who received standard-dose induction adalimumab. PUCAI response at week 8 was reported in 32 (68\%) of 47 patients who received high-dose induction adalimumab and $15(50 \%)$ of 30 patients who received standard-dose induction adalimumab (appendix p 13). In the mITT-E population at week 52, PUCAI remission was reported in $18(58 \%)$ of 31 patients who received high-dose maintenance adalimumab and by 14 (45\%) of 31 patients who received standard-dose maintenance adalimumab. PUCAI response at week 52 was reported in 16 (52\%) of 31 patients who received high-dose maintenance adalimumab and 18 (58\%) of 31 patients who received standard-dose maintenance adalimumab (appendix p 13).

In the subgroup analyses, a higher percentage of patients who were TNF inhibitor-naive versus patients who had previously received TNF inhibitors met the coprimary endpoints (appendix p 6). Of the patients in the mITT-E population who had disease flare during the maintenance period, FMS response at week 52 was reported in two (33\%) of six patients who received a reinduction dose and two (22\%) of nine patients without reinduction dose.

Clinically meaningful improvements in caregivers' WPAI absenteeism, presenteeism, work impairment, and activity impairment were shown in the mITT-E population by a decrease in mean scores from baseline starting at week 1 (when patients were still receiving induction treatment) and sustained to week 52 (figure 3). Clinically meaningful reductions in stool frequency and rectal bleeding Mayo subscores were observed in the mITT-E population as early as week 1 (when patients were still receiving induction treatment), which continued to decrease over time up to week 52 (figure 4).

Improvement in patient quality of life was shown in the mITT-E population by an increase in the mean total IMPACT III score from baseline starting at week 8 , which was sustained up to week 52 (appendix p 14). Patients had some evidence of reduced linear growth rate at baseline (mean height velocity $\mathrm{z}$ score $-0 \cdot 843$ ). Clinically meaningful changes from baseline to weeks 26 and 52 in z scores for height velocity were observed in the mITT-E population, and the largest BMI increase from baseline was seen over time in patients who received high-dose maintenance adalimumab group (appendix p 14).

In the integrated ITT population, mean adalimumab concentrations at weeks 2 and 4 were approximately twice as high in patients who received high-dose induction adalimumab than in those who received standard-dose induction adalimumab; this difference was smaller by week 8 (appendix p 7). During the maintenance period, adalimumab concentrations in those who received high-dose maintenance adalimumab were approximately two to three times higher than in those who received standard-dose maintenance adalimumab (appendix $\mathrm{p}$ 7). In patients who received high-dose induction adalimumab and were randomly assigned to receive either high-dose or standard-dose maintenance adalimumab, no differences in mean adalimumab concentration were observed by remission status during the maintenance period (appendix p 15). Three (3\%) of 100 patients were anti-

\begin{tabular}{|c|c|c|c|c|}
\hline & $\begin{array}{l}\text { Adalimumab } \\
\text { induction } \\
\text { period }^{*}(n=93)\end{array}$ & $\begin{array}{l}\text { Adalimumab } \\
\text { maintenance } \\
\text { period } \dagger(n=63)\end{array}$ & $\begin{array}{l}\text { Adalimumab } \\
\text { after flare } \ddagger \\
(n=22)\end{array}$ & $\begin{array}{l}\text { Any adalimumab } \\
\text { treatment } \$ \\
(n=93)\end{array}$ \\
\hline Adverse events & $52(56 \%)$ & $42(67 \%)$ & $9(41 \%)$ & $73(78 \%)$ \\
\hline Serious adverse events & $10(11 \%)$ & $9(14 \%)$ & $2(9 \%)$ & $21(23 \%)$ \\
\hline $\begin{array}{l}\text { Adverse events leading to } \\
\text { discontinuation }\end{array}$ & $3(3 \%)$ & $2(3 \%)$ & 0 & $5(5 \%)$ \\
\hline $\begin{array}{l}\text { Adverse events with reasonable } \\
\text { possibility of being related to } \\
\text { study drug }\end{array}$ & $12(13 \%)$ & $18(29 \%)$ & $4(18 \%)$ & $30(32 \%)$ \\
\hline $\begin{array}{l}\text { Serious adverse events with } \\
\text { reasonable possibility of being } \\
\text { related to study drug }\end{array}$ & $4(4 \%)$ & $1(2 \%)$ & 0 & $5(5 \%)$ \\
\hline Infection & $20(22 \%)$ & $23(37 \%)$ & $5(23 \%)$ & $44(47 \%)$ \\
\hline Serious infection & 0 & $5(8 \%)$ & 0 & $5(5 \%)$ \\
\hline Death & 0 & 0 & 0 & 0 \\
\hline
\end{tabular}

Data are $n$ (\%). *Treatment-emergent adverse events during the induction period are shown, and were defined as events with an onset date on or after the first dose date of adalimumab in the induction period, up to 70 days after the last dose date of adalimumab in the induction period, and before the first dose date of adalimumab in the maintenance period (if applicable). †Treatment-emergent adverse events during the maintenance period are shown, and were defined as events with an onset date on or after the first dose date of adalimumab in the maintenance period and before rerandomisation due to first disease flare, if applicable, and up to 70 days after the last dose date of adalimumab in the maintenance period; events with an onset date on or after the first dose date in the long-term extension study were excluded; safety population restricted to patients with at least one dose of adalimumab during the maintenance period. 抽eatment-emergent adverse events after disease flare are shown, and were defined as events with an onset date on or after the first dose date of the study drug after rerandomisation due to first disease flare and up to 70 days after the last dose date of the study drug; the events with an onset date on or after the first dose date in the long-term extension study were excluded; the safety population was restricted to patients who received at least one dose of adalimumab during the maintenance period who had at least one flare during the maintenance period. STreatment-emergent adverse events during any adalimumab treatment throughout the study are shown, and were defined as events with an onset date on or after the first dose date of adalimumab and up to 70 days after the last dose date of adalimumab and before the first dose date in the long-term extension study (if applicable), whichever came first; for patients who received placebo during the maintenance period, the treatment-emergent adverse event collection period ended 70 days after last induction dose of adalimumab and restarted with their next adalimumab dose (if applicable)

Table 2: Overview of adalimumab treatment-emergent adverse events of interest in the safety population and subpopulations 
adalimumab antibody-positive during the study. Serum adalimumab concentrations were higher in patients who were anti-adalimumab antibody-negative than in those who were anti-adalimumab antibody-positive. However, there was no apparent effect of anti-adalimumab antibodies on the efficacy or safety of adalimumab in these patients (two tested anti-adalimumab antibodypositive at week 26 and one at week 52; all three responded at week 8 and two were in remission at week 8 and 52; none had disease flare).

In the safety population, $52(56 \%)$ of 93 of patients reported one or more treatment-emergent adverse events, and $10(11 \%)$ patients reported one or more treatmentemergent serious adverse events during the induction period (table 2). Throughout any adalimumab exposure in the study, $73(78 \%)$ patients had one or more treatmentemergent adverse events, with $21(23 \%)$ patients reporting one or more treatment-emergent serious adverse event. Most serious adverse events were not related to treatment and did not lead to discontinuation from the study (table 2). Overall, five (5\%) patients had adverse events with reasonable possibility of being study-drug related that resulted in drug interruption (four patients; headache and loss of consciousness $[\mathrm{n}=1]$; injection site inflammation $[n=1]$; cytomegalovirus enterocolitis $[n=1]$; and asceptic meningitis and malaise, arthralgia, hypoaesthesia, and paraesthesia $[\mathrm{n}=1]$ ) or drug withdrawal (pericarditis $[\mathrm{n}=1]$ ).

The most common adverse events were headache, anaemia, and ulcerative colitis flare during the induction period and ulcerative colitis flare, headache, and nasopharyngitis during the maintenance period (appendix p 8). No deaths, malignancies, tuberculosis, or demyelinating diseases were reported. Two (2\%) of 93 patients had a colectomy. 13 (14\%) of 93 patients were hospitalised due to ulcerative colitis during the induction period, and six $(8 \%)$ of 74 patients were hospitalised during the maintenance period. Safety results were consistent in the integrated safety population (appendix p 9) and in Japanese patients. Treatment-emergent adverse events, serious adverse events, and adverse events leading to discontinuation were slightly higher in patients who received standard-dose induction adalimumab versus those who received high-dose induction adalimumab; however, the number of patients who had an event was low, and these results should be interpreted with caution.

\section{Discussion}

To date, ENVISION I is the largest phase 3 study of a biologic therapy in paediatric patients with moderate-to-severe ulcerative colitis. 77 (83\%) of 93 children with moderate-to-severe ulcerative colitis were PMS responders at week 8 . 74 (80\%) children continued to receive adalimumab as maintenance therapy and had clinically meaningful rates of remission (29-45\%), including corticosteroid-free remission (31\%), response (61-68\%), and mucosal healing (39-52\%) at week 52. Mayo score-based efficacy measures were supported by PUCAI clinical remission and response at weeks 8 and 52. Furthermore, improvements in quality of life, BMI, growth rate, stool frequency, rectal bleeding Mayo subscores, and caregivers' work productivity were observed with adalimumab. The safety profile was consistent with that of previous studies and the established safety profile of adalimumab. ${ }^{17,18,21-23}$ Results were consistent in the integrated analysis populations, which included patients from the Japanese substudy. Overall, these results suggest that adalimumab therapy is an effective, safe, and well tolerated treatment option for children with moderate-to-severe ulcerative colitis.

To our knowledge, adalimumab is the first subcutaneously administered, self-injectable biologic treatment option approved for paediatric ulcerative colitis. Infliximab is the only other biologic therapy approved for paediatric ulcerative colitis ${ }^{15}$ and requires infusions. ${ }^{12,13}$ Infliximab showed efficacy in children with moderate-to-severe ulcerative colitis in an open-label phase 3 study. ${ }^{24}$ At week 8 , clinical response was reported in $44(73 \%)$ of 60 patients and clinical remission by $24(40 \%)$ of 60 (based on Mayo score) or 17 (33\%) of 51 (based on PUCAI). Overall, 45 patients who were responders were randomly assigned to maintenance infliximab treatment at week 8 , and PUCAI remission was reported in eight (38\%) of 21 patients at week 54 with infliximab $5 \mathrm{mg} / \mathrm{kg}$ every 8 weeks. ${ }^{24}$ In the pooled adalimumab group of ENVISION I, PUCAI remission at week 8 was reported in $42 \%$ of patients, and PUCAI remission at week 52 was reported in $52 \%$ of patients.

The colectomy rate at year 1 was low in ENVISION I (only two [2\%] patients); by comparison, five (8\%) of 60 patients required colectomy in the infliximab study. ${ }^{24}$ The cumulative colectomy rate in patients with paediatriconset ulcerative colitis varies between studies, with 5-year reported rates of $15-26 \%$. ${ }^{3,25}$

In the original study design of ENVISION I, patients were to be randomly assigned (2:2:1) to receive high-dose maintenance adalimumab, standard-dose maintenance adalimumab, or placebo. However, because of enrolment issues due to non-acceptance of the placebo group, ${ }^{26}$ the study design was changed (M11-290 Protocol Amendment 4, Nov 2, 2017) to cease random assignment into the placebo group and to compare coprimary and ranked secondary maintenance endpoints with external adult placebo rates derived from a meta-analysis of eligible studies, as agreed with regulators. An external adult placebo control was also used in the infliximab study of children with ulcerative colitis..$^{24}$ Although use of external control data might have caveats because of differences in patient populations and other confounding factors, application of meta-analytic methods allows the use of external controls in a principled way. ${ }^{27}$ The clinical studies for the meta-analysis were selected based on their similarity with the ENVISION I study design (induction period followed by randomised withdrawal of active drug in 
induction responders in maintenance period as applicable), patient population (regarding previous and concomitant therapies, disease severity, and geographical region), and endpoint definitions and time points. In addition, the upper bound of the $95 \% \mathrm{CI}$ of the point estimates from the meta-analysis were conservatively used as the external placebo control rate. Extrapolation from adult trials for approved medications has been suggested for drug development in paediatric inflammatory bowel disease. ${ }^{28}$

In addition, the efficacy results of the coprimary endpoint at week 52 were based on centrally read endoscopies. ENVISION I also included a new rescue treatment option that evaluated reinduction dose in the case of flare, showing evidence of benefit without safety concerns; however, these results should be interpreted with caution because of the small number of patients. Although the study was not powered to compare the high-dose groups with the standard-dose groups, in general, high-dose adalimumab was numerically more efficacious than standard-dose adalimumab in the coprimary and ranked secondary endpoints (except for the last ranked endpoint: corticosteroid-free FMS remission at week 52 in week 8 PMS responders who were taking corticosteroids at baseline) during induction and maintenance periods, with absolute differences up to $16 \%$ noted between groups. Adalimumab concentrations were higher in the high-dose versus standarddose maintenance adalimumab group. However, mean adalimumab concentrations within the dose groups were similar regardless of remission status. In general, adalimumab concentrations in paediatric patients were similar to those in adult patients with ulcerative colitis receiving similar doses. ${ }^{29}$

No deaths, malignancies, active tuberculosis, or demyelinating diseases were observed in the study, and the safety profile was consistent with that of previous adalimumab studies in adults with ulcerative colitis, paediatric patients with Crohn's disease, and other indications. ${ }^{17,18,21}$ Our findings add to the extensive safety profile of adalimumab across multiple indications in adults $^{22}$ and children. ${ }^{23}$

The limitations of this study include a low number of patients evaluated and an uneven regional distribution of patients (most were from eastern Europe). In addition, because of non-acceptance of internal placebo, the primary efficacy analysis used external adult placebo controls for comparison. Strengths of this study included individualised dosing of adalimumab per body weight in a specific paediatric population with moderate-to-severe ulcerative colitis, objective assessment of eligibility and outcome due to central reading of endoscopies, and daily electronic capture of patient-reported outcomes and high-quality monitoring of data.

Clinically meaningful rates of clinical remission and response, including steroid-free remission and mucosal healing, were reported in children with moderate-to-severe ulcerative colitis who received adalimumab. No new safety signals were observed, suggesting that adalimumab is an efficacious and safe treatment option for children with moderate-to-severe ulcerative colitis.

\section{Contributors}

All authors were involved in the conception of the study, data analysis and interpretation, and manuscript writing. All authors were involved in the editing and revision of the manuscript. All authors had access to the data. NMC, MB, and AL verified the underlying data. All authors approved the final version of the manuscript.

\section{Declaration of interests}

NMC reports speaker fees from AbbVie; advisory board fees from AbbVie and Eli Lilly; research funding from AbbVie, Eli Lilly, Shire, Takeda, Janssen, and Pfizer; and writing support from AbbVie. WAF reports consulting fees from Boehringer Ingelheim, Takeda, AbbVie, Janssen, and Robarts. JK reports consultation fees from Nestle; research grants from Nutricia; and honoraria from Bristol Myers Squibb, Nestle, Nutricia. FMR reports grants and research support from Nestlé, AbbVie, MSD, and Janssen; served as a member of advisory boards for Centocor (DEVELOP), AbbVie (CAPE and LEA), MSD France (SAC), Nestlé, Danone, Mead Johnson, Nutricia, Takeda, Celgene, Biogen, Shire, Pfizer, and Therakos; and reports payment and honorarium for lectures from AbbVie, Danone, Nutricia, and Nestlé. NMM, MV, TF-H, YSG, MB, and AL are full-time AbbVie employees and might own AbbVie stock and stock options. DT reports consultation fees and honoraria from Janssen, Pfizer, Ferring, AbbVie, Takeda, Biogen, Atlantic Health, Shire, Celgene, Lilly, Roche, ThermoFisher, Bristol Myers Squibb; research support from Janssen, Ferring, AbbVie, and Takeda; and royalties from Hospital for Sick Children. All other authors declare no competing interests.

\section{Data sharing}

AbbVie is committed to responsible data sharing regarding the clinica trials we sponsor. This includes access to anonymised, individual and trial-level data (analysis data sets), as well as other information (eg, protocols and clinical study reports), as long as the trials are not part of an ongoing or planned regulatory submission. This includes requests for clinical trial data for unlicensed products and indications. These clinical trial data can be requested by any qualified researchers who engage in rigorous, independent scientific research, and will be provided following review and approval of a research proposal and Statistical Analysis Plan and execution of a Data Sharing Agreement. Data requests can be submitted at any time and the data will be accessible for 12 months, with possible extensions considered. More information on the process is available online.

\section{Acknowledgments}

AbbVie funded the study, contributed to its design, and was involved in the collection, analysis, and interpretation of the data, and in the writing, review, and approval of the manuscript. Medical writing support was provided by Maria Hovenden (ICON, North Wales, PA, USA), which was funded by AbbVie. We thank Nisha Kwatra for contributing to the study as well as all the patients and their families who participated in the trial and the research staff at all the institutions who supported the study.

\section{References}

1 Bradley GM, Oliva-Hemker M. Pediatric ulcerative colitis: current treatment approaches including role of infliximab. Biologics 2012; 6: 125-34.

2 Romano C, Syed S, Valenti S, Kugathasan S. Management of acute severe colitis in children with ulcerative colitis in the biologics era. Pediatrics 2016; 137: e20151184.

3 Van Limbergen J, Russell RK, Drummond HE, et al. Definition of phenotypic characteristics of childhood-onset inflammatory bowel disease. Gastroenterology 2008; 135: 1114-22.

4 Sykora J, Pomahacova R, Kreslova M, Cvalinova D, Stych P, Schwarz J. Current global trends in the incidence of pediatric-onset inflammatory bowel disease. World J Gastroenterol 2018; 24: 2741-63.

5 Benchimol EI, Fortinsky KJ, Gozdyra P, Van den Heuvel M Van Limbergen J, Griffiths AM. Epidemiology of pediatric inflammatory bowel disease: a systematic review of international trends. Inflamm Bowel Dis 2011; 17: 423-39.
For more on the request process see https://www.abbvie.com/ our-science/clinical-trials/ clinical-trials-data-andinformation-sharing/data-andinformation-sharing-withqualified-researchers.htm 
6 Kim SC, Ferry GD. Inflammatory bowel diseases in pediatric and adolescent patients: clinical, therapeutic, and psychosocial considerations. Gastroenterology 2004; 126: 1550-60.

7 Burgess CJ, Henderson P, Jones GR, Lees CW, Wilson DC. Paediatric patients (less than age of 17 years) account for less than $1.5 \%$ of all prevalent inflammatory bowel disease cases. J Pediatr Gastroenterol Nutr 2020; 71: 521-23.

8 Boot AM, Bouquet J, Krenning EP, de Muinck Keizer-Schrama SM. Bone mineral density and nutritional status in children with chronic inflammatory bowel disease. Gut 1998; 42: 188-94.

9 Turner D, Ruemmele FM, Orlanski-Meyer E, et al. Management of paediatric ulcerative colitis, part 1: ambulatory care-an evidencebased guideline from European Crohn's and Colitis Organization and European Society of Paediatric Gastroenterology, Hepatology and Nutrition. J Pediatr Gastroenterol Nutr 2018; 67: 257-91.

10 Hommel KA, Denson LA, Crandall WV, Mackner LM. Behavioral functioning and treatment adherence in pediatric inflammatory bowel disease: review and recommendations for practice. Gastroenterol Hepatol 2008; 4: 785.

11 Beese SE, Harris IM, Dretzke J, Moore D. Body image dissatisfaction in patients with inflammatory bowel disease: a systematic review. BMJ Open Gastroenterol 2019; 6: e000255

12 Van Assche G, Vermeire S, Rutgeerts P. Management of loss of response to anti-TNF drugs: change the dose or change the drug? J Crohns Colitis 2008; 2: 348-51.

13 Guerra I, Bermejo F. Management of inflammatory bowel disease in poor responders to infliximab. Clin Exp Gastroenterol 2014; 7: 359-67.

14 Kennedy NA, Heap GA, Green HD, et al. Predictors of anti-TNF treatment failure in anti-TNF-naive patients with active luminal Crohn's disease: a prospective, multicentre, cohort study. Lancet Gastroenterol Hepatol 2019; 4: 341-53.

15 Janssen. Remicade (infliximab) full prescribing information. 2020. https://www.janssenlabels.com/package-insert/productmonograph/prescribing-information/REMICADE-pi.pdf (accessed May 26, 2021).

16 AbbVie. Humira (adalimumab) full prescribing information. 2021. https://www.rxabbvie.com/pdf/humira.pdf (accessed May 26, 2021).

17 Reinisch W, Sandborn WI, Panaccione R, et al. 52-week efficacy of adalimumab in patients with moderately to severely active ulcerative colitis who failed corticosteroids and/or immunosuppressants. Inflamm Bowel Dis 2013; 19: 1700-09.
18 Sandborn WJ, van Assche G, Reinisch W, et al. Adalimumab induces and maintains clinical remission in patients with moderateto-severe ulcerative colitis. Gastroenterology 2012; 142: 257-65.

19 Feagan BG, Rutgeerts P, Sands BE, et al. Vedolizumab as induction and maintenance therapy for ulcerative colitis. N Engl J Med 2013; 369: 699-710.

20 Sandborn WJ, Su C, Panes J. Tofacitinib as induction and maintenance therapy for ulcerative colitis. N Engl J Med 2017; 377: 496-97.

21 Hyams JS, Griffiths A, Markowitz J, et al. Safety and efficacy of adalimumab for moderate to severe Crohn's disease in children. Gastroenterology 2012; 143: 365-74.

22 Burmester GR, Gordon KB, Rosenbaum JT, et al. Long-term safety of adalimumab in 29,967 adult patients from global clinical trials across multiple indications: an updated analysis. Adv Ther 2020; 37: 364-80.

23 Horneff G, Seyger MMB, Arikan D, et al. Safety of adalimumab in pediatric patients with polyarticular juvenile idiopathic arthritis, enthesitis-related arthritis, psoriasis, and Crohn's disease. J Pediatr 2018; 201: 166-75.

24 Hyams J, Damaraju L, Blank M, et al. Induction and maintenance therapy with infliximab for children with moderate to severe ulcerative colitis. Clin Gastroenterol Hepatol 2012; 10: 391-99.

25 Rinawi F, Assa A, Eliakim R, et al. Risk of colectomy in patients with pediatric-onset ulcerative colitis. J Pediatr Gastroenterol Nutr 2017; 65: 410-15.

26 Turner D, Koletzko S, Griffiths AM, et al. Use of placebo in pediatric inflammatory bowel diseases: a position paper from ESPGHAN, ECCO, PIBDnet, and the Canadian Children IBD Network. J Pediatr Gastroenterol Nutr 2016; 62: 183-87.

27 Schmidli H, Haring DA, Thomas M, Cassidy A, Weber S, Bretz F. Beyond randomized clinical trials: use of external controls. Clin Pharmacol Ther 2020; 107: 806-16.

28 Turner D, Griffiths AM, Wilson D, et al. Designing clinical trials in paediatric inflammatory bowel diseases: a PIBDnet commentary. Gut 2020; 69: 32-41.

29 Colombel JF, Jharap B, Sandborn WJ, et al. Effects of concomitant immunomodulators on the pharmacokinetics, efficacy and safety of adalimumab in patients with Crohn's disease or ulcerative colitis who had failed conventional therapy. Aliment Pharmacol Ther 2017; 45: 50-62. 